\title{
Bean common mosaic virus Isolate Exhibits a Novel Pathogenicity Profile in Common Bean, Overcoming the $b c-3$ Resistance Allele Coding for the Mutated eIF4E Translation Initiation Factor
}

\author{
Xue Feng, James R. Myers, and Alexander V. Karasev
}

First and third authors: Department of PSES, University of Idaho, Moscow; second author: Department of Horticulture, Oregon State University, Corvallis; and third author: Bioinformatics and Computational Biology Program, University of Idaho, Moscow. Accepted for publication 10 July 2015.

\begin{abstract}
Feng, X., Myers, J. R., and Karasev, A. V. 2015. Bean common mosaic virus isolate exhibits a novel pathogenicity profile in common bean, overcoming the $b c-3$ resistance allele coding for the mutated eIF4E translation initiation factor. Phytopathology 105:1487-1495.

Resistance against Bean common mosaic virus (BCMV) in Phaseolus vulgaris is governed by six recessive resistance alleles at four loci. One of these alleles, $b c-3$, is able to protect $P$. vulgaris against all BCMV strains and against other potyviruses; $b c-3$ was identified as the $e I F 4 E$ allele carrying mutated eukaryotic translation initiation factor gene. Here, we characterized a novel BCMV isolate 1755a that was able to overcome $b c-2$ and $b c-3$ alleles in common bean. Thus, it displayed a novel pattern of interactions with resistance genes in $P$. vulgaris, and was assigned to a new pathogroup, PG-VIII. The IVT7214 cultivar supporting the

replication of BCMV-1755a was found to have the intact homozygous $b c-3$ cleaved amplified polymorphic sequences marker and corresponding mutations in the $e I F 4 E$ allele that confer resistance to BCMV isolates from all other pathogroups as well as to other potyviruses. The VPg protein of 1755a had seven amino acid substitutions relative to VPgs of other BCMV isolates unable to overcome $b c-3$. The 1755a genome was found to be a recombinant between NL1, US1 (both PG-I), and a yet unknown BCMV strain. Analysis of the recombination patterns in the genomes of NL1 and US1 (PG-I), NY15P (PG-V), US10 and RU1-OR (PG-VII), and 1755a (PG-VIII), indicated that P1/HC-Pro cistrons of BCMV strains may interact with most resistance genes. This is the first report of a BCMV isolate able to overcome the $b c-3$ resistance allele, suggesting that the virus has evolved mechanisms to overcome multiple resistance genes available in common bean.
\end{abstract}

Bean common mosaic virus (BCMV) is an important pathogen of common bean (Phaseolus vulgaris L.). It is transmitted by aphids in a nonpersistent manner, and can also be efficiently transmitted through seed (Flores-Estevez et al. 2003; Morales and Bos 1988; Singh and Schwartz 2010). The resistance to BCMV in common bean is governed by seven resistance alleles: one dominant $I$ allele and six recessive alleles $\left(b c-u, b c-1, b c-1^{2}, b c-2, b c-2^{2}\right.$, and $\left.b c-3\right)$ (Drijfhout 1978; Kelly et al. 1995). Two recessive alleles, $b c-u$ and $b c-3$, when present together even in the absence of the $I$ allele, confer broad resistance against BCMV strains, and against other potyviruses, like Bean common mosaic necrosis virus (BCMNV) and Clover yellow vein virus (ClYVV) (Drijfhout 1978; Drijfhout and Morales 2005; Hart and Griffiths 2013).

BCMV exists as a complex of strains that can be differentiated by their ability to overcome the individual recessive alleles or their combinations (Drijfhout 1978; Drijfhout and Morales 2005). Based on their biological responses on bean differential hosts carrying different combinations of these resistance genes, BCMV isolates are classified into seven pathogroups (PGs), numbered from I to VII (Drijfhout 1978; Drijfhout and Morales 2005; Larsen et al. 2005). BCMV is a member of the genus Potyvirus, family Potyviridae (Adams et al. 2011). It has a single-stranded, positive-sense RNA genome of ca. $10 \mathrm{~kb}$ excluding the $3^{\prime}$-terminal poly(A), with a covalently-linked VPg protein at the $5^{\prime}$-terminus (Adams et al.

Corresponding author: A. V. Karasev; E-mail address: akarasev@uidaho.edu

*The $\boldsymbol{e}$-Xtra logo stands for "electronic extra" and indicates that two supplementary tables are published online.

http://dx.doi.org/10.1094/PHYTO-04-15-0108-R

(C) 2015 The American Phytopathological Society
2011; Morales and Bos 1988). The BCMV genome encodes a single polyprotein which is cleaved cotranslationally and posttranslationally by three virus-specific proteases into 10 mature proteins (Adams et al. 2005). A single capsid protein (CP) encapsidates virus RNA forming filamentous particles of ca. 720 to $770 \mathrm{~nm}$ long and 12 to $15 \mathrm{~nm}$ wide (Morales and Bos 1988). Recently, BCMV isolates were found to display substantial genome sequence diversity (Feng et al. 2014a,b; Larsen et al. 2005; Martin et al. 2014; Naderpour et al. 2009). Specifically, several BCMV isolates related to the RU1 strain group revealed recombinant genomes, and these recombination events were hypothesized to result in biological changes shifting their pathotype specificity (Feng et al. 2014a,b). In particular, the genome region spanning the $\mathrm{P} 1$ and HC-Pro cistrons was hypothesized to be involved in interactions with the $b c-2^{2}$ gene in common bean, thus defining the pathotype VII of BCMV (Feng et al. 2014a,b). However, specific mechanisms facilitating interactions with most of the resistance genes and genetic determinants of BCMV involved in these interactions are not known.

The only allele with a known mechanism of resistance to BCMV in common bean is $b c-3$, identified as the eIF4E allele carrying a mutated eukaryotic translation initiation factor gene (Naderpour et al. 2010). A cleaved amplified polymorphic sequence (CAPS) marker cosegregates with the $b c-3$ allele; this polymorphism was due to a mutation in the $e I F 4 E$ allele (Naderpour et al. 2010). The mutated $e I F 4 E$ allele conferred resistance to BCMV and at least two other potyviruses, BCMNV and ClYVV (Hart and Griffiths 2013; Naderpour et al. 2010). The eIF4E allele-mediated recessive resistance has been studied for several potyviruses infecting pepper, lettuce, potato, cereals, and cucurbits (Abdul-Razzak et al. 2009; Borgstrom and Johansen 2001; Bruun-Rasmussen et al. 2007; Charron et al. 2008; Kang et al. 2005a; Kanyuka et al. 2005; Kuhne et al. 2003; Moury et al. 2004; Nicaise et al. 2003). Mutations in 
genes coding for translation initiation factors eIF4E and eIF(iso)4E resulted in multiple recessive resistance genes conferring effective resistance to potyviruses in both monocots and dicots (Kang et al. 2005b; Truniger and Aranda 2009; Wang and Krishnaswamy 2012).

In the common bean/BCMV pathosystem, $b c-3$ conferred complete resistance to all strains and pathotypes of BCMV as well as to BCMNV and ClYVV, when present in a homozygous state and in combination with the $b c-u$ helper allele (Drijfhout 1978; Drijfhout and Morales 2005; Hart and Griffiths 2013; Naderpour et al. 2010). Because of its ability to confer complete, strain nonspecific resistance against BCMV and other legume-infecting potyviruses, the $b c-3$ allele has been introduced by breeders into common bean germplasm to protect different market classes of dry and snap beans against BCMV (Drijfhout 1978; Hart and Griffiths 2013; Kelly et al. 1995; Singh and Schwartz 2010).

Here, we describe the unique field isolate BCMV-1755a collected in the Willamette Valley, OR, in the summer of 2013. Comparison of the biological, serological, and molecular properties of this isolate with other known BCMV isolates suggested that, unlike any known BCMV strain, BCMV-1755a can overcome both the $b c-2$ and $b c-3$ alleles, and, thus, represents a new pathogroup, PG-VIII. The genome of BCMV-1755a as well as genomes of the two reference isolates, US1 from PG-I, and NY15P from PG-V, were found to be recombinant with extended sections of the genome having homologous sequences. This is the first report of a BCMV isolate able to overcome the $b c-3$ resistance allele.

\section{MATERIALS AND METHODS}

Virus sources and maintenance. All reference BCMV and BCMNV isolates used in this work originated from the USDA-ARS Prosser, WA, collection, and were described previously (Feng et al. 2014a,b). A field isolate of BCMV, named 1755a, was collected in Corvallis, OR, from the field-grown common bean accession 91-1755 exhibiting symptoms of mosaic and leaf deformation. This line is one of 59 accessions that were collected by Mike Dickson (Professor Emeritus, Cornell University) in China in 1991 and deposited in the USDA National Plant Germplasm System (NPGS) plant introduction system in 1995 where it has been stored informally. These accessions were first grown in the field at Oregon State University in 2009. No virus symptoms were observed in 911755 at that time, but several other accessions exhibited classic BCMV mosaic mottle symptoms. When the collection was grown again in 2012, severe virus symptoms were observed in a number of lines, suggesting that the virus had spread during the initial grow out. After confirmation that isolate 1755a displayed BCMVspecific reactivity in ELISA, it was subjected to further characterization at the Plant Virology Laboratory at University of Idaho. All virus isolates were propagated in the bean 'Dubbele Witte' using mechanical inoculation and then maintained under greenhouse conditions. BCMV isolates US1, NY15P, and 1755a, and the $\mathrm{BCMNV}$ isolate TN1 were propagated and purified using the purification protocol described previously (Feng et al. 2014b).

Biological and serological characterization. The biological characterization of all three isolates on a set of bean differentials (Drijfhout 1978) was performed as described previously (Feng et al. $2014 \mathrm{a}, \mathrm{b})$. This set of bean differentials represented the various host groups (HG) of common bean and included (Table 1) cultivars 'Dubbele Witte' (DW, HG 0), 'Stringless Green Refugee' (SGR, HG 1), 'Redlands Greenleaf C' (RGLC, HG 2), 'Redlands Greenleaf B' (RGLB, HG 3), 'Sanilac' (HG 4), UI 35 (HG 6), 'IVT7214' (HG 7), 'Jubila' (HG 9), 'Amanda' (HG 10), 'US1006' (HG 11), and 'IVT7233' (HG 11). Three reference isolates, BCMV-US1 (PG-I), BCMV-NY15P (PG-V), and BCMNV-TN1 (PG-VI), were included in this analysis as controls. All eleven bean differential lines carrying different resistance gene combinations (Table 1) were mechanically inoculated with each isolate (three plants per cultivar), and plants were placed in the greenhouse with standard summer-time growth conditions (16-h day photoperiod and daytime/nighttime temperatures of $25 / 20^{\circ} \mathrm{C}$ ). Symptoms were recorded at 4 weeks postinoculation, and concomitantly, samples were collected for triple-antibody sandwich (TAS)-enzyme-linked immunosorbent assay (ELISA) testing as described elsewhere (Feng et al. 2014b). Experiments were repeated three times.

Cloning strategy, sequencing, and sequence analysis. For all three isolates, 1755a, US1, and NY15P, the whole-genome cloning strategy, sequencing, and sequence analysis were conducted as described previously (Feng et al. 2014b). Briefly, isolates were subjected to a reverse transcription-polymerase chain reaction (RT-PCR) amplification with degenerate primers targeting the HCPro, CI, and NIb-CP regions (Ha et al. 2008), which were amplified and sequenced after AT-cloning. These initial sequenced contigs were later linked through additional RT-PCR assays using specific primers from the three contigs, followed by cloning and sequencing using primer-walking; the 5'-terminal sequence was determined through the use of the $5^{\prime}$-RACE approach as described previously (Feng et al. 2014b). The RNA extraction, RT-PCR, cloning, and sequencing steps were performed according to the protocol described in Feng et al. (2014b). All primers used in this study for RT-PCR amplifications and sequencing are listed in Supplementary Table S1. The complete viral genomes were assembled using SeqMan (DNASTAR, Madison, WI). The sequences for NY15P, US1, and 1755a genomes have been deposited in the GenBank database and will appear under the accession numbers KT175568, KT175569, and KT175570, respectively. All sequences were initially analyzed using the BLASTn 2.2.17 (Altschul et al. 1997) tool available at the National Center for Biotechnology Information (NCBI). Open reading frames (ORFs) were identified using the ORF Finder program available at NCBI. Complete sequences of $\mathrm{BCMV}$ isolates were aligned using ClustralX Ver. 2.0 (Conway

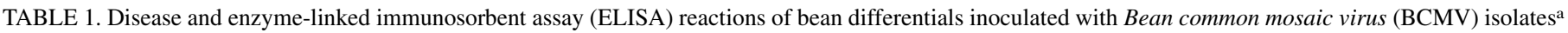

\begin{tabular}{|c|c|c|c|c|}
\hline Bean cultivar & Resistance genes & US1 (PG-I) & NY15P (PG-V) & 1755a (PG-VIII) \\
\hline 'Dubbele Witte' & none & $+/+$ & $+/+$ & $+/+$ \\
\hline 'Stringless Green Refugee' & $i, b c-u$ & $+/+$ & $+/+$ & $+/+$ \\
\hline 'Redlands Greenleaf C' & $i, b c-u, b c-1$ & $-1-$ & $+/+$ & $-1-$ \\
\hline 'Redlands Greenleaf B' & $i, b c-u, b c-1^{2}$ & $-1-$ & $-1-$ & $-1-$ \\
\hline 'Sanilac' & $i, b c-u, b c-2$ & $-1-$ & $+/+$ & $+/+$ \\
\hline 'UI35' & $i, b c-u, b c-1^{2}, b c-2^{2}$ & $-1-$ & $-1-$ & $-1-$ \\
\hline 'IVT7214' & $i, b c-u, b c-2, b c-3$ & $-1-$ & $-1-$ & $+/+$ \\
\hline 'Jubila' & $I, b c-1$ & $-1-$ & $-1-$ & $-1-$ \\
\hline 'Amanda' & $I, b c-1^{2}$ & $-1-$ & $-1-$ & $-1-$ \\
\hline 'US1006’ & $I, b c-u, b c-2^{2}$ & $-1-$ & $-1-$ & $-1-$ \\
\hline 'IVT7233' & $I, b c-u, b c-1^{2}, b c-2^{2}$ & $-1-$ & $-1-$ & $-1-$ \\
\hline
\end{tabular}

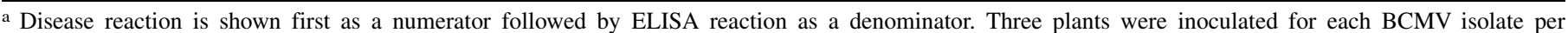
experiment; numerator: + = symptoms on inoculated beans; - = no symptoms on inoculated beans; denominator: + designates ELISA signal (A 405 ) in an infected plant exceeding healthy control 10-fold or more; - designates ELISA signal in an infected plant equal to that of a healthy control. 
Institute, UCD, Dublin). Further analysis was conducted with the Recombination Detection Program v.4.16 (RDP4) (Martin et al. 2005).

gDNA extraction, CAPS marker eIF4E-RsaI analysis, and eIF4E sequencing. Genomic DNA (gDNA) was extracted from $0.1 \mathrm{~g}$ of young leaf tissue using the CTAB methodology as described in Naderpour et al. (2010). For CAPS analysis, a 541-bp fragment of $e I F 4 E$ was amplified by PCR (Naderpour et al. 2010), using $1 \mu \mathrm{l}$ of gDNA extract in a $25-\mu \mathrm{l}$ reaction volume containing Taq-buffer (Genscript, Piscataway, NJ), $0.4 \mathrm{mM}$ of each of the oligonucleotide primers, $0.2 \mu \mathrm{M}$ ENM-FWe and ENM-RVe primers (Naderpour et al. 2010), and 1.25 units of Taq DNA polymerase (Genscript). Amplification included 30 cycles: 20 s of denaturation at $94^{\circ} \mathrm{C}, 20 \mathrm{~s}$ of annealing at $55^{\circ} \mathrm{C}$ and $35 \mathrm{~s}$ of elongation at $72^{\circ} \mathrm{C}$ in a thermal cycler (Mastercycler S-Pro, Eppendorf) after an initial denaturation at $95^{\circ} \mathrm{C}$ for $2 \mathrm{~min}$. Eight microliters of PCR product was subsequently subjected to RsaI (New England Biolabs, Ipswich, MA) digestion in a $20-\mu$ l reaction volume and analyzed by agarose gel electrophoresis.

The 541-bp fragments amplified from the gDNA extracted from bean cultivars 'SGR' and 'IVT'7214' were cloned into the T-Easy plasmid vector (Promega, Madison, WI). Three independent clones for each recombinant plasmid were selected and subjected to sequencing at the Genewiz laboratory (South Plainfield, NJ) from both ends of the cloned insert using plasmid-specific primers. The sequences were assembled using SeqMan (DNASTAR, Madison, WI) and analyzed using the BLASTn 2.2.17 (Altschul et al. 1997) tool available at the NCBI. Sequences of eIF4E were aligned using ClustralX Ver. 2.0 (Conway Institute, UCD, Dublin). The partial sequences of the eIF4E genes for 'SGR' and 'IVT7214' were deposited in the GenBank database under accession numbers KT175571 and KT175572, respectively.

\section{RESULTS}

BCMV-1755a is serotype B isolate that overcomes $b c-2$ and $\boldsymbol{b} \boldsymbol{c}-3$ alleles. When screened on the 11 bean differentials, 1755a, US1, and NY15P, induced typical mosaic mottle, raised dark green blistering, leaf deformation, and often growth retardation in susceptible differentials. Based on the pathogenicity profiles exhibited on differentials of our two control isolates, US1 was classified as belonging to PG-I (as expected), while NY15P was classified as belonging to PG-V (as expected; Table 1). Isolate $1755 \mathrm{a}$, on the other hand, was able to infect 'Dubbele Witte', 'Stringless Green Refugee', 'Sanilac', and 'IVT7214' exhibiting a novel and unusual pathogenicity profile (Table 1). In particular, its ability to replicate in 'IVT7214' was quite unexpected. Symptoms induced by $1755 \mathrm{a}$ in 'IVT7214' were visible at 3 to 4 weeks postinoculation as mild mosaic, mild leaf deformations, and slight downward leaf curling (Fig. 1A). Visual symptoms of BCMV infection were confirmed by laboratory diagnosis using BCMVspecific TAS-ELISA, with the $\mathrm{OD}_{405}$ signal for infected plants exceeding uninfected controls at least 10 -fold (Fig. 1B). Isolate 1755a replicating in 'IVT7214' was also used as inoculum for reinoculations of bean differentials and found to exhibit the same stable pathogenicity profile infecting 'Dubbele Witte', 'Stringless Green Refugee', 'Sanilac', and 'IVT7214'.

'IVT7214' carries the $b c-3$ allele, in addition to $b c-u$ and $b c-2$ alleles, and hence is considered immune to all known BCMV strains (Drijfhout 1978; Drijfhout and Morales 2005; Hart and Griffiths 2013). Based on this pathogenicity profile, it can be concluded that 1755 a overcomes both $b c-2$ and $b c-3$ resistance alleles in $P$. vulgaris (Table 1), even in the presence of the $b c-u$ helper allele, and hence represents a novel BCMV pathogroup, which we have designated pathogroup VIII following the convention established for the BCMV pathotypes (Drijfhout 1978; Drijfhout and Morales 2005).

Isolate 1755 a was subjected to a serological characterization with TAS-ELISA side-by-side with reference isolates US1 (B-serotype),
NY15P (B-serotype), US10 (B-serotype), and TN1 (A-serotype), in order to determine its serotype. All four isolates were captured on the ELISA plate using either anti-TN1, or anti-US10 rabbit antiserum, and were subsequently probed with two different detecting antibodies as described previously (Feng et al. 2014b). The anti-US10 antiserum reacted to all BCMV isolates tested, and also, as expected, to the BCMNV isolate TN1, when used as the detecting antibody. On the other hand, the anti-TN1 antiserum reacted only with the homologous isolate TN1 when used as the detecting antibody. This serological reactivity profile (Supplementary Table S2) suggested that isolate 1755a displayed the

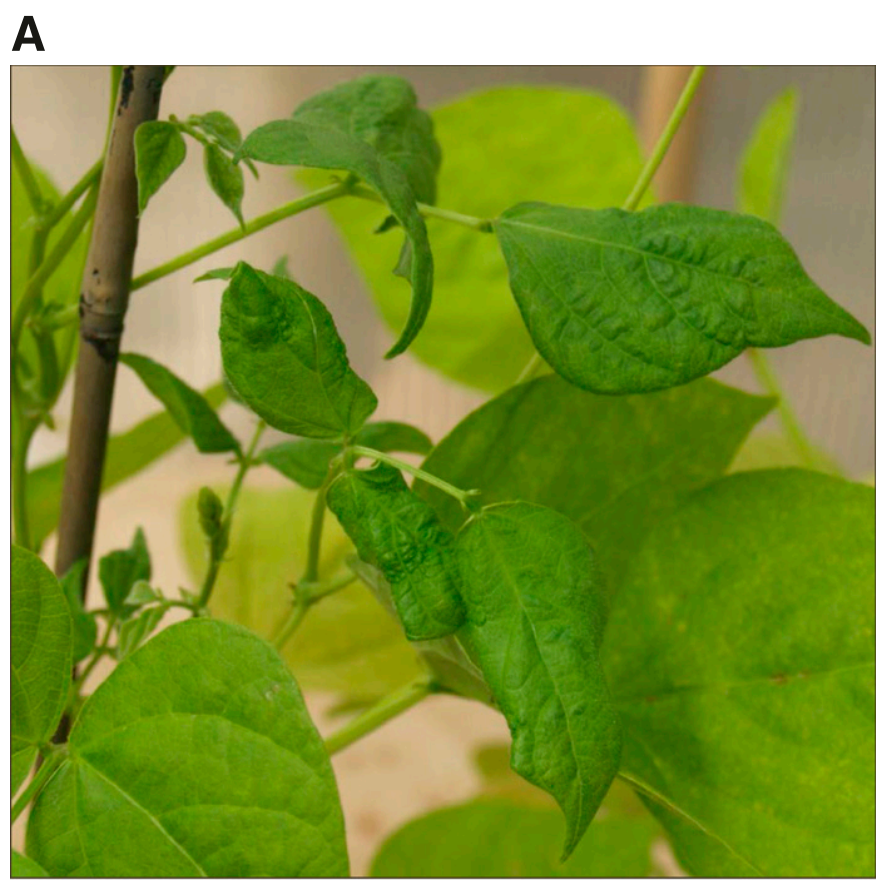

B

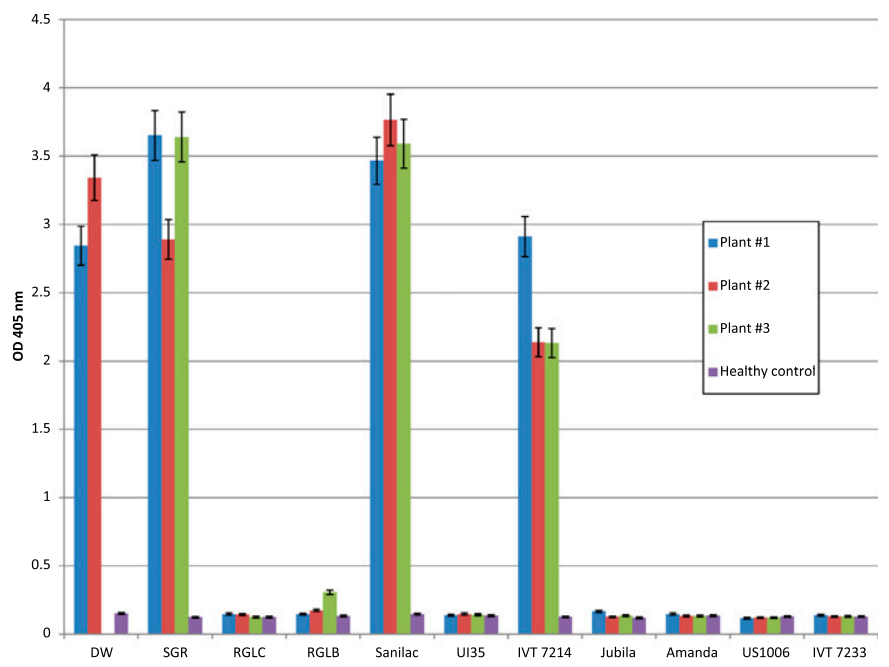

Fig. 1. A, Mild mosaic, and leaf deformations induced upon inoculation of Bean common mosaic virus (BCMV) isolate 1755a in 'IVT7214', 3 weeks postinoculation (wpi). B, An example of the triple-antibody sandwich (TAS)enzyme-linked immunosorbent assay (ELISA) assessment of BCMV-1755a infection in a set of bean differentials (Table 1), 4 wpi. Three individual plants per cultivar were infected with isolate $1755 \mathrm{a}$ and one plant was left uninfected; for 'Dubbele Witte' (DW), two plants were inoculated in this experiment. Vertical bars represent averages of two duplicate samples for the same plant analyzed by TAS-ELISA in the same experiment. Samples were considered positive if the $\mathrm{OD}_{405}$ signal for the infected plant exceeded the one for an uninoculated plant threefold. 
B-serotype, also characteristic of the control isolates US1 and NY15P (Berger et al. 1997; Feng et al. 2014a,b; Mink and Silbernagel 1992; Vetten et al. 1992).

IVT 7214 has the CAPS marker for $b c-3$, and carries the mutated eIF4E allele associated with resistance to potyviruses. In order to determine, if 'IVT7214' used in our experiments harbored the $b c-3$ gene, we analyzed the presence of the $b c-3$ specific CAPS marker as described by Naderpour et al. (2010). Two $P$. vulgaris genotypes were chosen for comparison: susceptible SGR, carrying only the $b c$ - $u$ allele, and 'IVT7214' carrying both $b c-u$ and $b c-3$ genes. For each cultivar, genomic DNA was used as a template for CAPS marker DNA amplification. Using the primer pair ENM-FWe/RVe, PCR fragments of $541 \mathrm{bp}$ were amplified from both cultivars. Digestion with RsaI of 'IVT7214' PCR products cleaved the 541-bp fragment into 381- and 160-bp fragments, as would be expected in $b c-3$ carrying, homozygous genotypes (Fig. 2A). The PCR products derived from SGR were not cleaved by $R s a$ I (Fig. 2A), as would be expected for a susceptible cultivar. To confirm if the mutations in the $e I F 4 E$ gene involved in the disruption of the interactions between eIF4E and the VPg, were present in the 'IVT7214' eIF4E gene, these 541-bp PCR fragments were cloned and sequenced for both 'SGR' and 'IVT7214' cultivars. After the intron sequence removal, the partial coding sequence of a fragment derived from SGR (408-bp) was $99 \%$ identical to the published partial sequence of the $e I F 4 E$ gene of 'IVT7214' (KC417369.1) available in GenBank, while the same partial coding sequence of a fragment amplified from 'IVT7214' (411 bp) was $100 \%$ identical to the same published sequence of the eIF4E gene of 'IVT7214' (KC417369.1).

These partial coding eIF $4 E$ gene sequences from both genotypes, 'SGR' and 'IVT7214', were aligned (Fig. 2B), and known mutations involved in expression of the eIF4E-mediated resistance to BCMV and other potyviruses (Hart and Griffiths 2013; Naderpour et al. 2010) were analyzed. One-codon deletion and four codon differences leading to amino acid substitutions were found between 'SGR' and 'IVT7214' in this partial coding sequence: del/Thr (position 32), Asn/Lys (126), Phe/Tyr (161), Ala/Glu (194), and Asp/Gly (299). The nucleotide substitution at position 161 (T/A) introduced the RsaI cleavage site into the 'IVT7214' sequence. Except for the deletion of an entire codon in the SGR sequence at nucleotide 32, all other nucleotide changes observed in the eIF4E coding sequences were consistent with data published by Naderpour et al. (2010) and Hart and Griffiths (2013). Hence, it was confirmed that 'IVT7214' used in our host range tests had the intact CAPS marker for the $b c-3$ gene, and carried the homozygous, mutated $e I F 4 E$ allele associated with resistance to BCMV and other potyviruses.

BCMV-1755a has a recombinant genome carrying large sections homologous with isolates NY15P, NL1, and US1. Initially, small genome fragments from the HC-Pro, CI, and NIb/CP regions of $\mathrm{BCMV}$ isolate $1755 \mathrm{a}$ were amplified using RT-PCR with degenerate primers (Ha et al. 2008), and sequenced using the approach described by Feng et al. (2014b). Two of these three initial clones, $\mathrm{CI}$ and $\mathrm{NIb} / \mathrm{CP}$, were $98 \%$ identical to the known sequence of the BCMV strain NL1, while the HC-Pro clone sequence was only $92 \%$ identical to the NL1 sequence. Gaps between the three initial clones were filled in via RT-PCR amplification using specific primers as listed in supplementary Table S1. The very $5^{\prime}$-terminal sequences for isolate 1755a were obtained using 5'-RACE (Feng et al. 2014b). Upon sequence assembly, 1755a genome was found to be 10,064-nt long, excluding the poly (A) and, based on conceptual translation, encoded a single polyprotein of 3,222 aa. The $1755 \mathrm{a}$ sequence was compared with the known BCMV genomes which revealed that BCMV-1755a whole genome sequence shared the closest similarity to several sequences deposited in GenBank as NL1 strain (e.g., accession number AY112735; 94\% nucleotide identity).

TABLE 2. Recombination patterns of select Bean common mosaic virus isolates detected by recombination-detecting programs

\begin{tabular}{lcc}
\hline Isolate & Recombination breakpoint(s) & $P$ value $^{\mathrm{b}}$ \\
\hline NL1 & 2124 & $3.95 \times 10^{-27}$ \\
NL1 & 6717 & $3.95 \times 10^{-27}$ \\
NY15P & 2124 & $3.95 \times 10^{-27}$ \\
NY15P & 6717 & $3.95 \times 10^{-27}$ \\
NY15P & 9381 & $3.06 \times 10^{-31}$ \\
US10 & 2832 & $1.87 \times 10^{-167}$ \\
1755a & 2169 & $4.39 \times 10^{-80}$ \\
1755a & 6680 & $4.83 \times 10^{-10}$ \\
\hline
\end{tabular}

a Breakingpoints indicate positions in the alignment of all sequences.

b Greatest $P$ value among recombinants identified by the recombinationdetecting programs RDP, GENECONV, Bootscan, Maxchi, Chimaera, SiScan, and 3Seq in RDP v4.16.

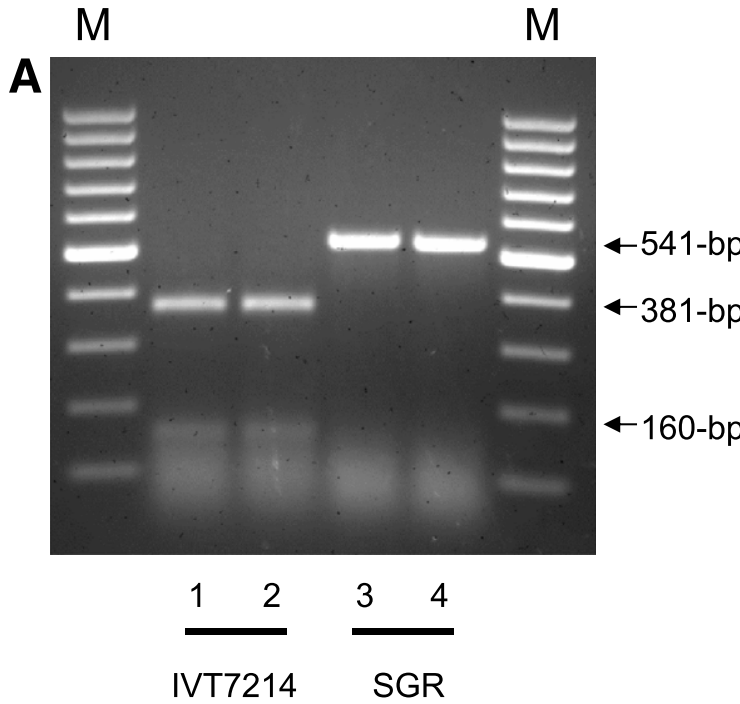

B SGR SGR IVT7214 91 ACCGATGAGCAAAACCCTAGCAGGGTCGACA---ACGACGACGATCTCGAAGATGGGGAGATCCTCGAAGATGCCGACGACGCCGCCTCC 87

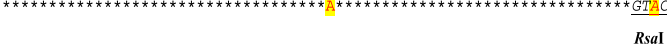
$\coprod^{\mathrm{Ala} / \mathrm{Glu}}$ SGR 178 TCCAAACAAGCCGCATGGGGCAGTTCCATCCGACCCATATACACTTTCTCCACCGTCGAAGAGTTTTGGAGCATTTACAATAACATTCAC 267

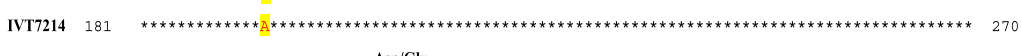
$\stackrel{\text { Asp/Gly }}{\square}$ SGR 268 CACCCGAGCAAGTTGGGTGTGGGGGCGGACTTTCACTGCTTCAAGCATAAGATTGAGCCCAGGAAAATGGACTATGACGTTCCAAAGGGG 357 IVT7214 271 SGR 358 GAAATCCGATACCAGTTGGTTG 408 IVT7214 $361 \quad * * * * * * * * * * * * * * * * * * * * * * \quad 411$

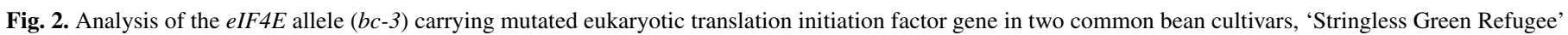

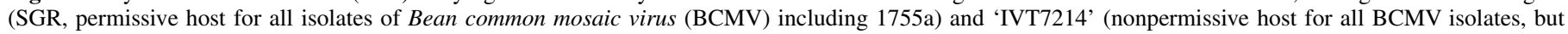

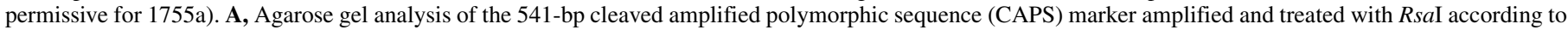

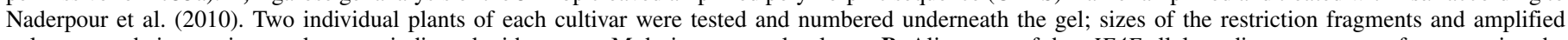

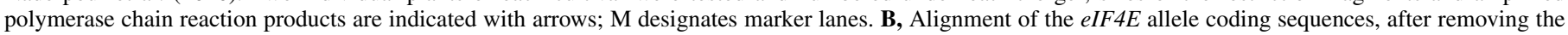

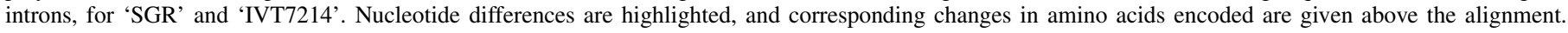
Position of the RsaI restriction site in 'IVT7214' used for the CAPS marker is underlined. 
The whole genomes of BCMV isolates US1 and NY15P were cloned and sequenced, using the same approach (Feng et al. 2014b). Upon sequence assembly, the US1 genome was found to be 10,052nt long, excluding the poly (A) and, based on conceptual translation, encoded a single polyprotein of 3,221 aa. NY15P genome was found to be 10,053-nt long, excluding the poly (A) and encoded a single polyprotein of 3,222 aa. Initially, the sequences of both isolates were compared with the known BCMV and BCMNV genomes, which revealed that the US1 and NY15P sequences shared the closest similarity to the isolate NL1 (PG-I, accession number AY112735; 94 and 97\% nucleotide identity to US1 [PG-I] and NY15P [PG-V], respectively).

The whole genomes for US1 (accession number KT175569), and NL1 (accession number AY112735) both from PG-I, together with NY15P (accession number KT175568, PG-V), were aligned using CLUSTALX and further analysis was conducted with the RDP4 program package (Table 2). Figure $3 \mathrm{~A}$ shows the comparison of all three sequences using the manual distance plot analysis, with the full-length NY15P sequence as the reference (Fig. 3A). Based on the RDP4 analysis, the $5^{\prime}$-terminal sequences of isolates US1 and NL1, between nucleotide 1 to 2124 , shared more similarities to each other (98\% identity) than to NY15p isolate (96\% identity). The sequences downstream between nucleotide 2125 to 6717 (position in alignment) in the US1 and NY15P genomes were quite similar (97\% identity), while NL1 had $89 \%$ similarity to the US1 and NY15P sequences in this segment. The $3^{\prime}$-terminal genome segments between nucleotide 6718 to 9381 (position in alignment), were very similar among all three isolates (97 to $98 \%$ identity). In the 3 '-terminal region, between nucleotide 9382 to 10055 (position in alignment), sequences of US1 and NL1 were similar to each other (98\% identity) while NY15P shared only 91 to $92 \%$ identities with the two other sequences. It appears that genomes of isolates NL1 (PG-I) and NY15P (PG-V) on one hand, and isolate US1 (PG-I) on the other hand, carry long sequences between nucleotide 2125 to 6717 that are quite different, while most of the other areas of the genomes for all three are much closer. This indicates a possible recombination event leading to these similarity patterns (Fig. 3A).

To view these possible recombination patterns in the more global context of known BCMV whole genome sequences, we compared the whole genome sequences of US1, NY15P, and 1755a with the genomes of other BCMV isolates with known pathogenicity profiles (Table 2), in addition to NL1 (isolates from PG-VII; Feng et al. 2014a,b). This comparison conducted using the distance plot program from the RDP4 package is presented in Figure 3B. A large section of the US1 genome, from nucleotide 2833 to 10055 (the 3 '-terminus) was homologous between isolates US1 (PG-I) and US10 (PG-VII), suggesting that this large area is not involved in the pathogenicity determinants for either PG-I or PG-VII. A smaller section of the genome, between nucleotide 6718 to 9381 was homologous between isolates NL1 (PG-I), NY15P (PG-V), and 1755a (PG-VIII) (Fig. 3B). On the other hand, the two areas mentioned above, nucleotide 1 to 2124 and 9382 to 10055 were the least similar between US1/NL1 and NY15P sequences (Fig. 3A), suggesting that pathogenicity determinants for PG-I and PG-V may be located in these two regions. Unexpectedly, the BCMV-1755a genome had a large section, nucleotide 2170 to 10055 , which was homologous to the corresponding section of the NL1 genome (97\% identity), and a $5^{\prime}$-terminal section, nucleotide 1 to 2169 substantially different from all other BCMV genomes analyzed (Fig. 3B and C). These recombination patterns resulted in a complicated mosaic pattern presented on Figure 3C, which suggests that the P1 and HC-Pro cistrons as largely responsible for interactions with multiple BCMV resistance genes in common bean.

The VPg protein of the BCMV-1755a isolate has only seven amino acid substitutions relative to other BCMV isolates unable to overcome $\boldsymbol{b} \boldsymbol{c}-\mathbf{3}$. Since the $\mathrm{VPg}$ protein was implicated in interactions with the $b c-3$ gene in common bean (Hart and Griffiths 2013; Naderpour et al. 2010), sequences of several VPg proteins from BCMV strains unable to replicate in 'IVT7214' (NL1, US1, US10, RU1-OR, and NY15P) were aligned and compared with the VPg sequence of BCMV-1755a. The amino acid sequences of VPg proteins were identified based on the cleavage sites described for BCMV polyproteins by Wylie and Jones (2011). The N-terminal cleavage site used was VTTQ/G, the C-terminal cleavage site used was VAVE/S (VGIE/S for RU1-OR). Only seven positions out of 190 amino acids were unique for the BCMV-1755a isolate overcoming the $b c-3$ gene (Fig. 4), and distinct from other isolates unable to overcome the $b c-3$ gene. Four out of these seven differences were located very close to the N-terminus of the VPg protein: position $2(\mathrm{R} / \mathrm{K})$, position $3(\mathrm{~N} / \mathrm{K})$, position $5(\mathrm{~K} / \mathrm{M})$, and position $8(\mathrm{R} / \mathrm{K})$. Two of these substitutions, at positions 3 and 5 , resulted in chemically distinct amino acids that could change/disrupt the normal folding of a protein. The other three amino acid substitutions were scattered along the protein and located at position $55(\mathrm{~K} / \mathrm{R})$, position $115(\mathrm{~T} / \mathrm{K})$, and position 157 (S/A); only position 115 changed the chemical nature of the amino acid.

\section{DISCUSSION}

The genetic diversity characteristic of potyviruses is driven by mutation and recombination (Gibbs and Ohshima 2010; Nagy 2008; Roossinck 2003). This vast diversity allows potyviruses to adapt to new evolutionary niches, including their ability to overcome multiple resistance genes in different hosts (Gibbs and Ohshima 2010; Karasev and Gray 2013). One of the six recessive resistance alleles that govern interactions of BCMV isolates with common bean, $b c-3$ was considered the best resistance gene conferring broad, strain nonspecific resistance against all isolates of BCMV and against other potyviruses, e.g., BCMNV and ClYVV, in common bean (P. vulgaris L.) when present in a homozygous form with the $b c-u$ helper allele (Drijfhout 1978; Hart and Griffiths 2013; Kelly et al. 1995), and was identified as the $e I F 4 E$ allele coding for the eIF4E translation initiation factor (Hart and Griffiths 2013; Naderpour et al. 2010). Isolate BCMV-1755a described here is the first BCMV isolate breaking $b c-3$ mediated resistance in common bean, its characterization allows the examination of possible BCMV genetic determinants interacting with the host eIF4E allele.

Identification or narrowing down of the genetic determinants of BCMV interacting with all seven resistance alleles found in common bean is complicated at the moment due to the high genetic diversity of BCMV and relative scarcity of information about BCMV isolates from different pathogenicity groups; only some of them had been characterized (Feng et al. 2014a,b; Larsen et al. 2005; Martin et al. 2014; Naderpour et al. 2010).

Here, we continued our comparative genomic studies for BCMV strains from three different pathogenicity groups, PG-I (strain US1), PG-V (NY15P), and PG-VIII (1755a). PG-I comprises BCMV isolates unable to overcome any of the recessive alleles, $b c-1, b c-1^{2}$, $b c-2, b c-2^{2}$, or $b c-3$ in the presence of the effector gene $b c-u$. PG-V comprises isolates that are able to overcome recessive alleles $b c-1$ and $b c-2$, but not others. Interestingly, sequences determined for US1 (PG-I) and for NY15P (PG-V) were relatively similar to each other and to another BCMV isolate from PG-I, NL1 (Fig. 3A and B). US1 represented a recombinant between NL1 and another, yet unknown parent (Fig. 3A). Based on the sequence analysis of NY15P, and six other complete genomes of BCMV isolates with defined pathogenicity (Fig. 3B), the most likely genome areas involved in interactions with genes $b c-1$ and $b c-2$ may be located between positions 1 to 2124 or positions 9382 to 10055 . The newly established PG-VIII comprises the 1755a isolate which is able to overcome $b c-2$ and $b c-3$ recessive alleles in the presence of the effector gene $b c-u$, but not others. The recombination analysis of 1755a (Fig. 3B and C) suggested that the unique sequence region between positions 1 to 2169 may be responsible for the interactions 

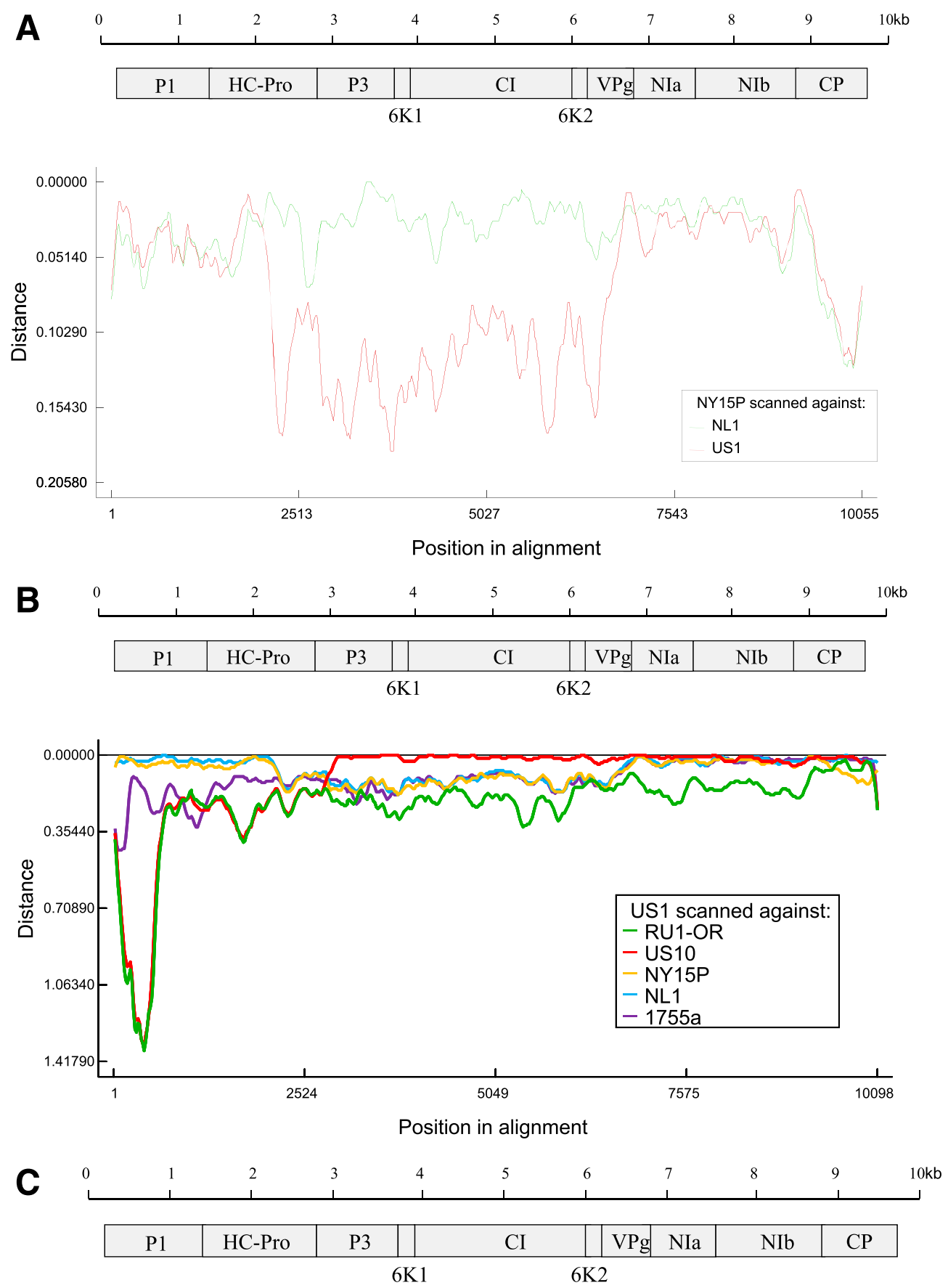

Pathotype I
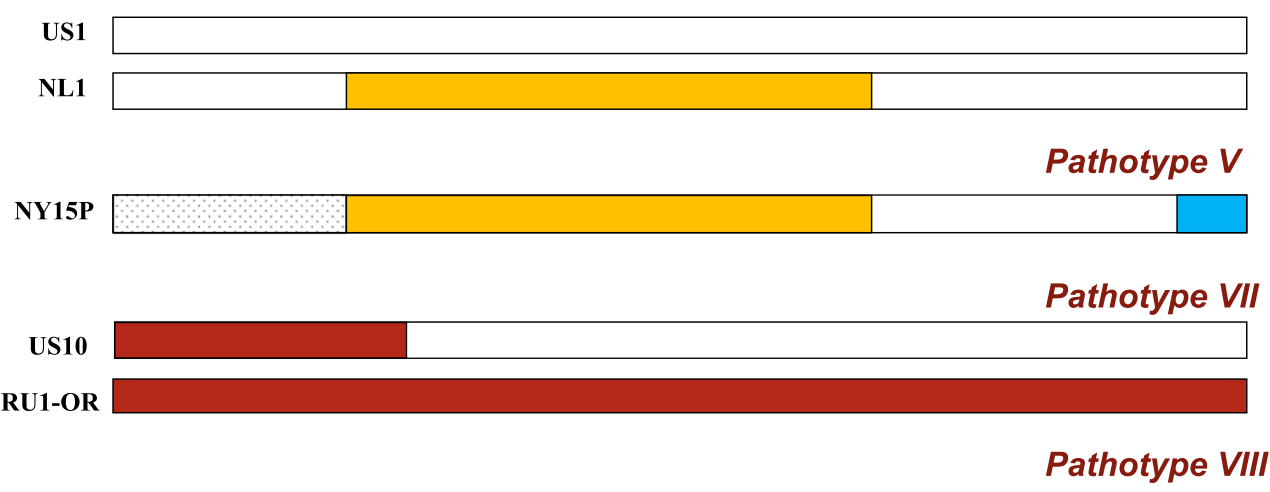

$1755 a$

$\square$ BCMV-US1 $\square$ BCMV-RU1-OR 
with $b c-2$ and/or $b c-3$ alleles; the overlapping region, nucleotide 1 to 2124 was defined as a possible determinant of the $b c-2$ interaction based on the analysis of the NY15P sequence (see above).

The high level of genetic diversity characteristic of BCMV (Feng et al. 2014a,b; Flasinski et al. 1996; Larsen et al. 2005; Zhou et al. 2014; this work) that resulted in distinct evolutionary lineages comprising common bean, peanut, and soybean isolates of BCMV (Zhou et al. 2014) suggests successful adaptation and specialization of the virus to several leguminous hosts. In common bean, BCMV was recently found to undergo an extensive recombination related to the ability of BCMV recombinants to overcome multiple resistance genes incorporated into common bean cultivars (Feng et al. 2014a,b). Since molecular mechanisms involved in the expression of BCMV resistance in common bean are unknown for most of these resistance genes, with the $b c$-3 allele being the only exception (Hart and Griffiths 2013; Naderpour et al. 2010), recombinants may provide some clues as to where the molecular determinants of the resistance may reside in the BCMV genome (Feng et al. 2014a,b).
$1755 a$

NL1

US 1

US 10

RU1 - OR

NY15P

$1755 a$

NL1

US 1

US 10

RU1 - OR

NY15P

$$
\begin{aligned}
& |\ldots| \ldots|\ldots| \ldots|\ldots| \ldots|\ldots| \ldots|\ldots| \ldots|\ldots| \ldots|\ldots| \ldots|\ldots| \\
& 1 \quad 11 \quad 21 \quad 31 \quad 41 \quad 51
\end{aligned}
$$

GRNRKL QRLKFRDAFD RKVGREVYAD DYTMEHTFGE AYTKKGKQKG STKTKGMGKK GKKRML QKLKFRDAFD RKVGREVYAD DYTMEHTFGE AYTKKGKQKG STKTKGMGRK GKKRML QKLKERDAFD RKVGREVYAD DYTMEHTFGE AYTKKGKQKG STKTKGMGRK GKKRML QKLKFRDAFD RKVGREVYAD DYTMEHTFGE AYTKKGKQKG STKTKGMGRK GKKRMM QKLKFRDAFD RKVGREVYAD EYTMEHTFGE AYTKKGKQKG STKTKGMGRK GKKRML QKLKFRDAFD RKVGREVYAD DYTMEHTFGE AYTKKGKQKG STKTKGMGRK

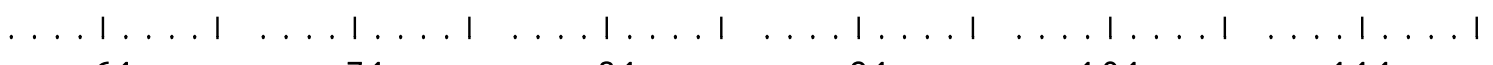
$\begin{array}{llllll}61 & 71 & 81 & 91 & 101 & 111\end{array}$ TRKFIHMYGV EPENYSMIRF VDPLTGATLD EGTRVDIRLV QEEFGEIRRQ KIDADELDTE TRKFIHMYGV EPENYSMIRF VDPLTGATLD EGTRVDIRLV QEEFGEIRRQ KIDDNELDKE TRNFIHMYGV EPENYSMVRF VDPLTGATLD EGTRVDIRLV QEEFGEIRKE KINDGELDKE TRNEIHMYGV EPENYSMIRF VDPLTGATLD EGTRVDIRLV QEGFGEIRKK KINDDELDKE TRNFIHMYGV EPENYSMIRF VDPLTGATLD EGTRVDIRLV QEEFGEIRK్ KIDEDELDKE TRNFIHMYGV EPENYSMIRF VDPLTGATLD EGTRVDIRLV QEEFGEIRRQ KIDNDDELNKE

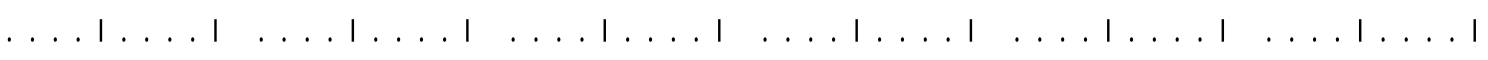

$$
\begin{aligned}
& \begin{array}{llllll}
121 & 131 & 141 & 151 & 161 & 171
\end{array}
\end{aligned}
$$

TVRNKPGIQA YFIGKNAEEA LKVDLTPHRP TLLCMNSNAI SGFPEREDEL RQTGLPVHIK TVRNKPGIQA YFIGKNAEEA LKVDLTPHRP TLLCMNSNAI AGFPEREDEL RQTGLPVHIK TVRRNPGIQA YFVGKNAEEA LKVDLTPHRP TLLCMNSNAI AGFPEREDEL RQTGLPVRIK TVIRNPGIQA YFVGKNAEEA LKVDLTPHRP TLLFMNSNAI AGFPEREDEL RQTGLPVRIK TVRRNPGIQA YFIGKNAEEA LKVDLTPHRP TLLCMNSNAI AGFPEREDEL RQTGLPVRIK TVRNKPGIQA YFIGKNAEEA LKVDLTPHRP TLLCMNSNAI AGFPEREDEL RQTGLPVRIK

$$
\ldots 1 \ldots 1 \ldots
$$

181

$1755 a \quad$ RSEVPEPNEE VAVE

NL1 RSEVPEPNEE VAVE

US1 RSEVPEPNEE VAVE

US10 RSEVPEPNEE VAVE

RU1-OR RSEVPEPSEE VGIE

NY15P RSEVPEPNEE VAVE

Fig. 4. Amino acid alignment of the VPg sequences for Bean common mosaic virus (BCMV)-1755a isolate, which is able to replicate in 'IVT7214', and several BCMV isolates unable to replicate in 'IVT7214', NL1, US1, US10, RU1-OR, and NY15P. Substitutions present only in BCMV-1755a are highlighted in light gray; substitutions that are not specific to BCMV-1755a are highlighted in dark gray.

Fig. 3. Recombination analysis of the two control Bean common mosaic virus (BCMV) isolates, US1 and NY15P and of the BCMV isolate 1755a, in comparison with the main BCMV strains. A, Manual distance plot based on the aligned full-length nucleotide sequences of BCMV isolates US1, NL1, and NY15P; NY15P (GenBank accession KT175568) was used as the reference strain. B, Manual distance plot based on the aligned full-length nucleotide sequences of BCMV isolates US1, NL1, NY15P, 1755a, US10, and RU1-OR; NL1 (GenBank accession AY112735) was used as the reference strain. $x$ axis represents nucleotide position in the alignment. $y$ axis represents relative distance from the reference sequence which is calculated using Kimura model (Kimura 1980). C, Schematic diagram showing putative BCMV recombination structures for BCMV isolates 1755a, RU1-OR, US1, NL1, and NY15P. The diagram reflects distance plot data presented in Figure 3. Similar shading of the rectangles indicates homologous sequences present in BCMV strains. 
Based on the molecular and biological analysis of three BCMV isolates, US1 (PG-I), NY15P (PG-V), and 1755a (PG-VIII), BCMV genome determinants interacting with genes $b c-1, b c-2$, and perhaps even $b c-3$ may reside in the $5^{\prime}$-terminal region of the genome coding for the P1 and HC-Pro proteins, between positions 1 to 2124 (Table 1; Fig. 3C), while a determinant involved in interactions with the $b c-1$ gene may also reside close to the $3^{\prime}$-terminus of the genome, nucleotide 9382 to 10055 (Table 1; Fig. 3C). Nevertheless, all these preliminary assignments will have to be confirmed through the reverse genetics experimentation.

Resistance breaking isolates have been described in several pathosystems that involve eIF4E/eIF(iso)4E recessive alleles and potyviruses (Truniger and Aranda 2009; Wang and Krishnaswamy 2012). Most of the examples of these resistance breaking potyvirus isolates had mutations in the virus VPg cistron identified as responsible for the resistance-breaking phenotype (Abdul-Razzak et al. 2009; Borgstrom and Johansen 2001; Bruun-Rasmussen et al. 2007; Charron et al. 2008; Kanyuka et al. 2005; Kuhne et al. 2003; Moury et al. 2004). Nevertheless, other potyvirus cistrons were also implicated in interactions with the $\operatorname{eIF} 4 E / e I F($ iso $) 4 E$ alleles, for instance the CI cistron in Lettuce mosaic virus isolates overcoming alleles $\mathrm{mol}^{1}$ and $\mathrm{mol}^{2}$ in lettuce (Abdul-Razzak et al. 2009), and the HC-Pro cistron in Potato virus A in both tobacco and potato (AlaPoikela et al. 2011). The mechanism of the resistance breaking of the eIF4E allele was proposed to involve the disruption of interactions between the eIF4E translation initiation factor and the VPg protein of potyviruses (Charron et al. 2008; Roudet-Tavert et al. 2007; Yeam et al. 2007). It is interesting that most of the mutations in the eIF4E proteins that confer resistance to different potyviruses were located in the cap-binding cavity of the eIF4E translation initiation factor, likely affecting interactions between eIF4E and VPg (Monzingo et al. 2007; Truniger and Aranda 2009; Yeam et al. 2007). Resistance breaking mutations in the VPg, on the other hand, were mostly found in the central region of the VPg thought to be directly interacting with the eIF4E protein (RoudetTavert et al. 2007). Of the seven amino acid substitutions found unique to the BCMV-1755a isolate VPg, four were clustered close to the N-terminus (Fig. 4). Precise identification of the residues in the BCMV-1755a VPg that might be involved in interactions with the mutated eIF4E protein will require reverse genetics and proteomics approaches.

From a practical perspective, finding of a BCMV isolate able to overcome both $b c-2$ and $b c-3$ alleles in common bean highlights the value of pursuing breeding material with multiple alleles conferring resistance to BCMV, even if perceived less effective, like $b c-1$ or $b c-1^{2}$. Isolates of BCMV with pathogenicity profiles similar to $1755 \mathrm{a}$ (PG-VIII) should be included in existing breeding programs as challenges, in search of other recessive genes conferring resistance to this novel pathotype of BCMV.

\section{ACKNOWLEDGMENTS}

We are grateful to A. R. Poplawsky and O. V. Nikolaeva for helpful discussions and valuable advice, to P. Berger, and P. Shiel for reference isolates US1 and NY15P of BCMV, and for TN1 isolate of BCMNV, and to J. Chojnacky and R. Nilsson for greenhouse and laboratory help. This work was funded in part through grants from the Idaho Bean Commission, the Idaho State Department of Agriculture, and by the Idaho Agricultural Experiment Station.

\section{LITERATURE CITED}

Abdul-Razzak, A., Guiraud, T., Peypelut, M., Walter, J., Houvenaghel, M. C., Candresse, T., Le Gall, O., and German-Retana, S. 2009. Involvement of the cylindrical inclusion $(\mathrm{CI})$ protein in the overcoming of an eIF4E-mediated resistance against Lettuce mosaic potyvirus. Mol. Plant Pathol. 10:109-113.

Adams, M. J., Antoniw, J. F., and Beaudoin, F. 2005. Overview and analysis of the polyprotein cleavage sites in the family Potyviridae. Mol. Plant Pathol. 6:471-487.
Adams, M. J., Zerbini, F. M., French, R., Rabenstein, F., Stenger, D. C., and Valkonen, J. P. T. 2011. Family Potyviridae. Pages 1069-1089 in: Virus Taxonomy. Ninth Report of the International Committee on Taxonomy of Viruses. A. King, M. Adams, E. Carstens, and E. Lefkowitz, eds. Elsevier, Oxford.

Ala-Poikela, M., Goytia, E., Haikonen, T., Rajamäki, M. L., and Valkonen, J. P. 2011. Helper component proteinase of the genus Potyvirus is an interaction partner of translation initiation factors eIF(iso)4E and eIF4E and contains a 4E binding motif. J. Virol. 85:6784-6794.

Altschul, S. F., Madden, T. L., Schaffer, A., Zhang, J., Zhang, Z., Miller, W., and Lipman, D. J. 1997. Gapped BLAST and PSI-BLAST: A new generation of protein database search programs. Nucleic Acids Res. 25: 3389-3402.

Berger, P. H., Wyatt, S. D., Shiel, P. J., Silbernagel, M. J., Druffel, K., and Mink, M. I. 1997. Phylogenetic analysis of the Potyviridae with emphasis on legume-infecting potyviruses. Arch. Virol. 142:1979-1999.

Borgstrom, B., and Johansen, I. E. 2001. Mutations in Pea seedborne mosaic virus genome-linked protein VPg alter pathotype-specific virulence in Pisum sativum. Mol. Plant-Microbe Interact. 14:707-714.

Bruun-Rasmussen, M., Moller, I. S., Tulinius, G., Hansen, J. K. R., Lund, O. S., and Johansen, I. E. 2007. The same allele of translation initiation factor 4E mediates resistance against two Potyvirus spp. in Pisum sativum. Mol. Plant-Microbe Interact. 20:1075-1082.

Charron, C., Nicolai, M., Gallois, J.-L., Robaglia, C., Moury, B., Palloix, A., and Caranta, C. 2008. Natural variation and functional analyses provide evidence for co-evolution between plant eIF4E and potyviral VPg. Plant J. 54:56-68.

Drijfhout, E. 1978. Genetic Interaction Between Phaseolus vulgaris and Bean common mosaic virus with Implications for Strain Identification and Breeding for Resistance. Centre for Agricultural Publication and Documents, Wageningen, The Netherlands.

Drijfhout, E., and Morales, F. 2005. Bean common mosaic. Pages 60-62 in: Compendium of Bean Diseases, 2nd ed. H. F. Scwartz, J. R. Steadman, R. Hall, and R. L. Forster, eds. American Phytopathological Society, St. Paul, MN.

Feng, X., Poplawsky, A. R., and Karasev, A. V. 2014a. A recombinant of Bean common mosaic virus induces temperature-insensitive necrosis in an I genebearing line of common bean. Phytopathology 104:1251-1257.

Feng, X., Poplawsky, A. R., Nikolaeva, O. V., Myers, J. R., and Karasev, A. V. 2014b. Recombinants of Bean common mosaic virus (BCMV) and genetic determinants of BCMV involved in overcoming resistance in common beans. Phytopathology 104:786-793.

Flasinski, S., Gunasinghe, U. B., Gonzales, R. A., and Cassidy, B. G. 1996. The cDNA sequence and infectious transcripts of peanut stripe virus. Gene 171:299-300.

Flores-Estevez, N., Acosta-Gallegos, J. A., and Silva-Rosales, L. 2003. Bean common mosaic virus and Bean common mosaic necrosis virus in Mexico. Plant Dis. 87:21-25.

Gibbs, A., and Ohshima, K. 2010. Potyviruses and the digital revolution. Annu. Rev. Phytopathol. 48:205-223.

Ha, C., Coombs, S., Revill, P. A., Harding, R. M., Vu, M., and Dale, J. L. 2008. Design and application of two novel degenerate primer pairs for the detection and complete genomic characterization of potyviruses. Arch. Virol. 153:25-36.

Hart, J. P., and Griffiths, P. D. 2013. A series of $e I F 4 E$ alleles at the $B c-3$ locus are associated with recessive resistance to Clover yellow vein virus in common bean. Theor. Appl. Biol. 126:2849-2863.

Kang, B.-C., Yeam, I., Frantz, D. J., Murphy, J. F., and Jahn, M. M. 2005a. The pvrl locus in pepper encodes a translation initiation factor eIF4E that interacts with tobacco etch virus VPg. Plant J. 42:392-405.

Kang, B.-C., Yeam, I., and Jahn, M. M. 2005b. Genetics of plant virus resistance. Annu. Rev. Phytopathol. 43:581-621.

Kanyuka, K., Druka, A., Caldwell, D., Tymon, A., McCallum, N., Waugh, R., and Adams, M. J. 2005. Evidence that the recessive bymovirus resistance locus rym 4 in barley corresponds to the eukaryotic translation initiation factor 4E gene. Mol. Plant Pathol. 6:449-458.

Karasev, A. V., and Gray, S. M. 2013. Continuous and emerging challenges of Potato virus $Y$ in potato. Annu. Rev. Phytopathol. 51:571-586.

Kelly, J. D., Afanador, L., and Haley, S. D. 1995. Pyramiding genes for resistance to Bean common mosaic virus. Euphytica 82:207-212.

Kimura, M. 1980. A simple method for estimating evolutionary rates of base substitutions through comparative studies of nucleotide sequences. J. Mol. Evol. 16:111-120.

Kuhne, T., Shi, N., Proeseler, G., Adams, M. J., and Kanyuka, K. 2003. The ability of a bymovirus to overcome the rym4-mediated resistance in barley correlates with a codon change in the VPg coding region on RNA1. J. Gen. Virol. 84:2853-2859.

Larsen, R. C., Miklas, P. N., Druffel, K. L., and Wyatt, S. D. 2005. NL-3 K strain is a stable and naturally occurring interspecific recombinant derived 
from Bean common mosaic necrosis virus and Bean common mosaic virus. Phytopathology 95:1037-1042.

Martin, D. P., Williamson, C., and Posada, D. 2005. RDP2: Recombination detection and analysis from sequence alignments. Bioinformatics 21:260-262.

Martin, K., Hill, J. H., and Cannon, S. 2014. Occurrence and characterization of Bean common mosaic virus strain NL1 in Iowa. Plant Dis. 98:1593.

Mink, G. I., and Silbernagel, M. J. 1992. Serological and biological relationships among viruses in the bean common mosaic virus subgroup. Arch. Virol. Suppl. 5:397-406.

Monzingo, A. F., Dhaliwal, S., Dutt-Chaudhuri, A., Lyon, A., Sadow, J. H., Hoffman, D. W., Robertus, J. D., and Browning, K. S. 2007. The structure of eukaryotic translation initiation factor-4E from wheat reveals a novel disulfide bond. Plant Physiol. 143:1504-1518.

Morales, F. J., and Bos, L. 1988. Bean common mosaic virus. Descriptions of Plant Viruses, No. 337. Association of Applied Biologists. http://www. dpvweb.net/dpv/showdpv.php?dpvno=337

Moury, B., Morel, C., Johansen, E., Guilbaud, L., Souche, S., Ayme, V., Caranta, C., Palloix, A., and Jacquemond, M. 2004. Mutations in Potato virus $Y$ genome-linked protein determine virulence toward recessive resistances in Capsicum annuum and Lycopersicon hirsutum. Mol. PlantMicrobe Interact. 17:322-329.

Naderpour, M., Lund, O. S., and Johansen, I. E. 2009. Sequence analysis of expressed cDNA of Bean common mosaic virus RU1 isolate. Iran J. Virol. 3:41-43.

Naderpour, M., Lund, O. S., Larsen, R., and Johansen, E. 2010. Potyviral resistance derived from cultivars of Phaseolus vulgaris carrying $b c-3$ is associated with the homozygotic presence of a mutated $e I F 4 E$ allele. Mol. Plant Pathol. 11:255-263.

Nagy, P. D. 2008. Recombination in plant viruses. Pages 133-156 in: Plant Virus Evolution. M. J. Roossinck, ed. Springer, Berlin, Heidelberg.

Nicaise, V., German-Retana, S., Sanjuán, R., Dubrana, M.-P., Mazier, M., Maisonneuve, B., Candresse, T., Caranta, C., and Le Gall, O. 2003. The eukaryotic translation initiation factor $4 \mathrm{E}$ controls lettuce susceptibility to the potyvirus Lettuce mosaic virus. Plant Physiol. 132:1272-1282.

Roossinck, M. J. 2003. Plant RNA virus evolution. Curr. Opin. Microbiol. 6: 406-409.

Roudet-Tavert, G., Michon, T., Walter, J., Delaunay, T., Redondo, E., and Le Gall, O. 2007. Central domain of a potyvirus VPg is involved in the interaction with the host translation initiation factor eIF4E and the viral protein HcPro. J. Gen. Virol. 88:1029-1033.

Singh, S., and Schwartz, H. F. 2010. Breeding common bean for resistance to diseases: A review. Crop Sci. 50:2199-2223.

Truniger, V., and Aranda, M. A. 2009. Recessive resistance to plant viruses. Adv. Virus Res. 75:119-159.

Vetten, H. J., Lesemann, D. E., and Maiss, E. 1992. Serotype A and B strains of Bean common mosaic virus are two distinct potyviruses. Arch. Virol. Suppl. 5:415-431.

Wang, A., and Krishnaswamy, S. 2012. Eukaryotic translation initiation factor 4E-mediated recessive resistance to plant viruses and its utility in crop improvement. Mol. Plant Pathol. 7:795-803.

Wylie, S. J., and Jones, M. G. 2011. The complete genome sequence of a Passion fruit woodiness virus isolate from Australia determined using deep sequencing, and its relationship to other potyviruses. Arch. Virol. 156: 479-482.

Yeam, I., Cavatorta, J. R., Ripoll, D. R., Kang, B.-C., and Jahn, M. M. 2007. Functional dissection of naturally occurring amino acid substitutions in eIF4E that confers recessive potyvirus resistance in plants. Plant Cell 19: 2913-2928.

Zhou, G.-C., Wu, X.-Y., Zhang, Y.-M., Wu, P., Wu, X.-Z., Liu, L.-W., Wang, Q., Hang, Y.-Y., Yang, J.-Y., Shao, Z.-Q., Wang, B., and Chen, J. Q. 2014. A genomic survey of thirty soybean-infecting Bean common mosaic virus (BCMV) isolates from China pointed BCMV as a potential threat to soybean production. Virus Res. 191:125-133. 